\title{
Branes at conical singularities and holography
}

\author{
B.S. Acharya, J. M. Figueroa-O'Farrill, C.M. Hull, B. Spence
}

\author{
Department of Physics \\ Queen Mary and Wesfield College \\ Mile End Road \\ London E1 4NS, UK \\ r.acharya,j.m.figueroa, c.m.hull,b.spence@qmw.ac.uk
}

\begin{abstract}
For supergravity solutions which are the product of an anti-de Sitter space with an Einstein space $X$, we study the relation between the amount of supersymmetry preserved and the geometry of $X$. Depending on the dimension and the amount of supersymmetry, the following geometries for $X$ are possible, in addition to the maximally supersymmetric spherical geometry: Einstein-Sasaki in dimension $2 k+1,3$-Sasaki in dimension $4 k+3$, 7-dimensional manifolds of weak $G_{2}$ holonomy and 6-dimensional nearly Kähler manifolds. Many new examples of such manifolds are presented which are not homogeneous and hence have escaped earlier classification efforts. String or $M$ theory in these vacua are conjectured to be dual to superconformal field theories. The brane solutions interpolating between these anti-de Sitter near-horizon geometries and the product of Minkowski space with a cone over $X$ lead to an interpretation of the dual superconformal field theory as the world-volume theory for branes at a conical singularity (cone branes). We propose a description of those field theories whose associated cones are obtained by (hyper-)Kähler quotients.
\end{abstract}

e-print archive: http://xxx.lanl.gov/abs/hep-th/9705006 


\section{Introduction and Motivation}

Maldacena [59] has conjectured that the 't Hooft large $N$ limit of $\mathcal{N}=4$ supersymmetric Yang-Mills with gauge group $\mathrm{SU}_{N}$ is dual to type IIB superstring theory on $\left(\operatorname{adS}_{5} \times S^{5}\right)_{N}$, where the subscript indicates that the sizes of the spaces grow with $N$. A more precise version of the conjecture was formulated in $[46,71]$ where a simple recipe was given for relating gauge theory correlators to string theory S-matrix elements, and these are given in terms of classical supergravity in the large $N$ limit.

This conjecture was motivated by considering $N$ parallel D3-branes for $N$ large and taking a limit in which the gauge theory on the brane decouples from the physics of the bulk [59]. When $g_{s} N$ is small (with $g_{s}$ the string coupling), the system is well-described by 4-dimensional super Yang-Mills theory with $\mathrm{SU}_{N}$ gauge group, while if $g_{s} N$ is large, the system is described by IIB string theory in the near-horizon geometry, which is $\operatorname{adS}_{5} \times S^{5}$. These are then dual descriptions of the same system, leading to the conjectured equivalence. String loop corrections correspond to $\frac{1}{N}$ corrections in the gauge theory so that in the large $N$ limit, one can use classical supergravity theory in the $\operatorname{adS}_{5} \times S^{5}$ background.

This can be generalised to any $p$-brane of superstring theory or $M$ theory and this leads to a relation between the worldvolume theory with $\mathrm{SU}_{N}$ gauge symmetry and the string or $M$ theory in the space-time which arises in the near-horizon limit of the $p$-brane spacetime [53]. Of particular interest are those cases - the D3-brane and the M2 and M5-branes - in which the nearhorizon geometry is a supersymmetric anti-de Sitter space solution of the form $\operatorname{adS}_{p+2} \times S^{d}(d \equiv D-p-2)$ and the worldvolume theory is a superconformal field theory. In these cases, there is a holographic duality between the string or $M$ theory in anti-de Sitter space and the superconformal field theory (which may be thought of as being at the boundary of the anti-de Sitter space $[59,71])$. The superconformal symmetry in $p+1$ dimensions is identified with the anti-de Sitter supersymmetry in $p+2$ dimensions.

An interesting extension of these ideas is to explore $p$-branes which exhibit near-horizon geometries of the form $\operatorname{ad}_{p+2} \times X_{d}$, where $X$ is an Einstein manifold. If the geometry is supersymmetric, then the string or $M$ theory in that background is expected to be holographically dual to a superconformal field theory. These vacua are not maximally supersymmetric unless $X$ is a round sphere or the real projective space, which is the near-horizon geometry of branes on an orientifold [72], and as a result the dual field theories have less than maximal supersymmetry. Many such cases have been studied already, particularly in the context of type IIB superstring theory, but also in $M$ 
theory [1]. A simple modification is to let $X=S^{d} / \Gamma$ be a finite quotient of the sphere $[54,58,63,6,2,36,26]$. Alternatively, if we recall that $S^{2 n+1}$ is a circle bundle over $\mathbb{C P}^{n}$, one can replace $\mathbb{C P}^{n}$ with other Kähler-Einstein $n$ folds. This was done in the type IIB context (i.e., $n=2$ ) in [55], generalising [64]. Another generalisation is to replace $S^{d} \cong \mathrm{SO}_{d+1} / \mathrm{SO}_{d}$ with another homogeneous space $G / H$. Homogeneous vacua of supergravity theories were studied intensively in the early days of Kaluza-Klein supergravity (see, e.g., [24]). There is a complete classification in dimension seven [17] and a partial list in dimension five [65]. One such five-dimensional example is $T^{1,1} \equiv$ $\left(\mathrm{SU}_{2} \times \mathrm{SU}_{2}\right) / \mathrm{U}_{1}$, whose dual conformal field theory was recently discussed by Klebanov and Witten [56] (see also [45]). They interpreted the vacuum in terms of the near-horizon geometry of parallel D3-branes at a conical singularity of a Calabi-Yau threefold. Similar ideas have been considered in [61].

The purpose of the present paper, which subsumes [27], is to study those $\mathrm{M}$ theory or superstring vacua of the form $\operatorname{adS}_{p+2} \times X_{d}$ that preserve some supersymmetry. Our aim will be to understand the constraints supersymmetry imposes on the geometry of the Einstein manifolds $X_{d}$, and then to use the geometry of these manifolds in order to identify the superconformal symmetries which are required under the adS/CFT correspondence. This is certainly likely to be useful in understanding generic features of this correspondence in supersymmetric cases.

Any solution of the form adS $\mathrm{S}_{p+2} \times X_{d}$ is the near-horizon geometry of a $p$-brane solution [23] which will be supersymmetric if the near-horizon geometry is, and which interpolates between $\operatorname{adS}_{p+2} \times X_{d}$ and a vacuum $M_{p+1} \times C(X)_{d+1}$ where $C(X)_{d+1}$ is a cone over $X$ (defined below) and $M_{p+1}$ is $(p+1)$-dimensional Minkowski space. The case in which $X_{d}$ (with $d=7$ ) is a coset space was considered in [14], but we will consider general Einstein spaces $X_{d}$ which in addition preserve some supersymmetries. The cone $C(X)$ is Ricci-flat and the number of supersymmetries of the $M_{p+1} \times C(X)_{d+1}$ vacuum depends on the number of covariantly constant spinors. This is determined by the holonomy group of $C(X)$, and such holonomies have been classified (see, e.g., [70]). The number of supersymmetries on $\operatorname{adS}_{p+2} \times X_{d}$ depends on the number of Killing spinors on $X$, but these all arise from covariantly constant spinors on $C(X)$ [4], leading to a characterisation of supersymmetric anti-de Sitter solutions.

We will show that for those vacua adS $\mathrm{S}_{p+2} \times X_{d}$ which are supersymmetric, the geometry of $X$ is highly constrained. Depending on $d \equiv D-p-2$, the possible geometries of $X$ are as follows: nearly Kähler for $d=6$, weak $G_{2}$ holonomy for $d=7$, Einstein-Sasaki for $d=2 k+1$, and 3-Sasaki for $d=4 k+3$; 
this gives many supersymmetric compactifications of string and $M$ theory which have not been considered previously.

Given a $p$-brane solution of the above type, the interpolating solution argument [59] then leads to a conjectured duality between the string or $M$ theory in a supersymmetric background of the form ad $\mathrm{S}_{p+2} \times X_{d}$ and a $(p+1)$ dimensional superconformal field theory on the worldvolume of $N$ coincident $p$-branes located at the conical singularity of the $M_{p+1} \times C(X)_{d+1}$ vacuum. The detailed description of the dual theories will be left to future investigations, and in this paper we will focus instead on generic features of the field theories which follow from the common properties of each of the geometries listed above.

This paper is organised as follows. In Section 2 we set the notation concerning the supersymmetric branes which we will study in this paper and we review the simple solutions with spherical near-horizon geometries. In Section 3 we discuss the solutions which can be interpreted as branes sitting at a conical singularity in a Ricci-flat manifold $C$, and we characterise the supersymmetric solutions in terms of the holonomy of $C$. In Section 4 we discuss their near-horizon geometries in detail. Section 5 contains many examples including those in the early Kaluza-Klein literature as well as some more recent ones which have hereto not been considered in relation with supergravity. As an application of our results, in Section 6 we describe how the near-horizon geometry induces the superconformal symmetry of the dual theory. Finally in Section 7 we offer some conclusions.

\section{Supersymmetric Branes and their Near-Horizon Geometries}

In this section we review the near-horizon geometries of the elementary brane solutions of string and $M$ theory. This section serves mostly to establish the notation.

\section{$2.1 \quad$ M-branes}

Eleven-dimensional supergravity consists of the following fields [62, 19]: a Lorentzian metric $g$, a closed 4 -form $F$ with a quantised flux and a gravitino $\Psi$. By a supersymmetric vacuum we will mean any solution of the equations of motion with $\Psi=0$ for which the supersymmetry variation $\delta_{\varepsilon} \Psi=0$, regarded as a linear equation on the spinor parameter $\varepsilon$, has nontrivial so- 
lutions. The eleven-dimensional spinorial representation is 32-dimensional and real, so there at most 32 linearly independent solutions. An important physical invariant of a supersymmetric vacuum is the fraction $\nu \equiv \frac{1}{32} n$ of the supersymmetry that the solution preserves. For example, $F=0$ and $g$ the flat metric on eleven-dimensional Minkowski spacetime is a supersymmetric vacuum with $\nu=1$; that is, it is maximally supersymmetric. Other maximally supersymmetric vacua are $\operatorname{adS}_{4} \times S^{7}$ and $\operatorname{adS}_{7} \times S^{4}$ with $\star F$ and $F$ having quantised flux on the $S^{7}$ and $S^{4}$, respectively.

Eleven-dimensional supergravity has four types of elementary solutions with $\nu=\frac{1}{2}$ : the M-wave [51] and the Kaluza-Klein monopole [43, 68, 52], and the elementary brane solutions: the M2-brane [25] and the M5-brane [47]. There should also be an M9-brane solution [52, 5]. In what follows we will focus on the M2- and M5-branes.

\subsubsection{The M2-brane}

The following background describes a number of parallel M2-branes [25]:

$$
\begin{aligned}
g & =H^{-\frac{2}{3}} g_{2+1}+H^{\frac{1}{3}} g_{8} \\
F & = \pm \operatorname{dvol}_{2+1} \wedge d H^{-1},
\end{aligned}
$$

where $g_{2+1}$ and dvol $_{2+1}$ are the metric and volume form on the three-dimensional Minkowskian worldvolume $\mathbb{E}^{2,1}$ of the branes; $g_{8}$ is the metric on the eight-dimensional euclidean space $\mathbb{E}^{8}$ transverse to the branes; and $H$ is a harmonic function on $\mathbb{E}^{8}$. For example, if we demand that $H$ depend only on the transverse radial coordinate $r$ on $\mathbb{E}^{8}$, we then find that

$$
H(r)=1+\frac{a^{6}}{r^{6}} ; \quad a^{6} \equiv 2^{5} \pi^{2} N \ell_{p}^{6}
$$

is the only solution with $\lim _{r \rightarrow \infty} H(r)=1$. This corresponds to $N$ coincident membranes at $r=0$. Here $\ell_{p}$ is the eleven-dimensional Planck length.

Other choices of $H$ are possible. For example one can consider multicentre generalisations, where $H(\boldsymbol{x})$ is an arbitrary harmonic function on $\mathbb{E}^{8}$ with pointlike singularities and suitable asymptotic behaviour $H(x) \rightarrow 1$, say, as $|\boldsymbol{x}| \rightarrow \infty$. This corresponds to parallel branes localised at the singularities of $H$. More generally, we can take $H$ to be invariant under some subgroup $G$ of isometries of $g_{8}$. These solutions correspond to branes which are 'delocalised' or smeared on the $G$-orbits.

We can easily determine the fraction $\nu$ of the supersymmetry which the above solution preserves [25]. The supersymmetry variation of the gravitino 
in a bosonic background $(g, F)$ is given by

$$
\delta_{\varepsilon} \Psi_{M}=\nabla_{M} \varepsilon-\frac{1}{288}\left(\Gamma_{M}^{P Q R S}-8 \delta_{M}^{P} \Gamma^{Q R S}\right) F_{P Q R S} \varepsilon,
$$

where $\nabla_{M}=\partial_{M}+\frac{1}{4} \omega_{M}{ }^{P Q} \Gamma_{P Q}$ is the spin connection. In the M2-brane Ansatz given by (1) and (2), equation (3) is solved by

$$
\varepsilon=H^{-\frac{1}{6}} \varepsilon_{\infty}
$$

where, choosing $x^{0}, x^{1}, x^{2}$ to be the directions tangent to the worldvolume of the brane, $\varepsilon_{\infty}$ obeys :

$$
\Gamma_{012} \varepsilon_{\infty}=\varepsilon_{\infty}
$$

Because $\Gamma_{012}$ squares to the identity and is traceless, we see that $\nu=\frac{1}{2}$. Nevertheless, the M2-brane interpolates between two maximally supersymmetric solutions: flat Minkowski space $\mathbb{E}^{10,1}$ infinitely far away from the brane, and $\operatorname{adS}_{4} \times S^{7}$ near the brane horizon [35, 21].

Indeed, notice that the metric on the transverse space is given by

$$
g_{8}=d r^{2}+r^{2} g_{S}
$$

where $g_{S}$ is the metric on the unit sphere $S^{7} \subset \mathbb{E}^{8}$. In the near-horizon region $r \ll a$,

$$
H(r) \sim \frac{a^{6}}{r^{6}}
$$

so that the membrane metric becomes

$$
g=a^{-4} r^{4} g_{2+1}+a^{2} r^{-2} d r^{2}+a^{2} g_{S} .
$$

The last term is the metric on a round $S^{7}$ of radius $a=\left(2^{5} \pi^{2} N\right)^{\frac{1}{6}} \ell_{p}$; whereas the first two combine to produce the metric on 4-dimensional anti-de Sitter spacetime with "radius" $R_{\mathrm{adS}}=\frac{1}{2} a$ :

$$
g_{\mathrm{adS}}=R_{\mathrm{adS}}^{2}\left[\frac{d u^{2}}{u^{2}}+\left(\frac{u}{R_{\mathrm{adS}}}\right)^{2} \frac{g_{2+1}}{R_{\mathrm{adS}}^{2}}\right]
$$

with $u=\frac{1}{4} r^{2} / R_{\text {adS }}$. 


\subsubsection{The M5-brane}

The M5-brane is the magnetic dual to the M2-brane. The background describing a number of parallel M5-branes is given by [47]:

$$
\begin{aligned}
g & =H^{-\frac{1}{3}} g_{5+1}+H^{\frac{2}{3}} g_{5} \\
F & = \pm 3 \star_{5} d H,
\end{aligned}
$$

where $g_{5+1}$ and dvol $_{5+1}$ are the metric and volume form on the six-dimensional Minkowskian worldvolume $\mathbb{E}^{5,1}$ of the branes; $g_{5}$ and $\star_{5}$ are the metric and Hodge operator on the five-dimensional euclidean space $\mathbb{E}^{5}$ transverse to the branes; and $H$ is a harmonic function on $\mathbb{E}^{5}$. For example, if we demand that $H$ depend only on the transverse radial coordinate $r$ on $\mathbb{E}^{5}$, we then find that

$$
H(r)=1+\frac{a^{3}}{r^{3}} ; \quad a^{3} \equiv \pi N \ell_{p}^{3}
$$

is the only solution with $\lim _{r \rightarrow \infty} H(r)=1$. This corresponds to $N$ coincident fivebranes at $r=0$.

As for the membrane solution, multicentre and 'delocalised' fivebrane solutions also exist.

The fraction $\nu$ of the supersymmetry which is preserved can be computed as before [47]. The gravitino shift equation in the bosonic background given by $(g, F)$ above is solved by

$$
\varepsilon=H^{-\frac{1}{12}} \varepsilon_{\infty}
$$

where, choosing $x^{0}, x^{1}, \ldots, x^{5}$ to be the directions tangent to the worldvolume of the fivebrane, $\varepsilon_{\infty}$ obeys

$$
\Gamma_{012345} \varepsilon_{\infty}=\varepsilon_{\infty} .
$$

$\Gamma_{012345}$ squares to the identity and is traceless, so that again $\nu=\frac{1}{2}$. Nevertheless, as for the M2-brane, the M5-brane also interpolates between two maximally supersymmetric solutions: flat Minkowski space $\mathbb{E}^{10,1}$ infinitely far away from the brane, and $\operatorname{adS}_{7} \times S^{4}$ near the brane horizon [35, 21].

Indeed,

$$
g_{5}=d r^{2}+r^{2} g_{S}
$$

where $g_{S}$ is now the metric on the unit sphere $S^{4} \subset \mathbb{E}^{5}$. In the near-horizon region $r \ll a$,

$$
H(r) \sim \frac{a^{3}}{r^{3}},
$$


so that the fivebrane metric becomes

$$
g \sim a^{-1} r g_{5+1}+a^{2} r^{-2} d r^{2}+a^{2} g_{S} .
$$

The last term is the metric on a round $S^{4}$ of radius $a=(\pi N)^{\frac{1}{3}} \ell_{p}$, whereas the first two combine to produce the metric on 7-dimensional anti-de Sitter spacetime with "radius" $R_{\mathrm{adS}}=2 a$, analogous to (5),

$$
g_{\mathrm{adS}}=R_{\mathrm{adS}}^{2}\left[\frac{d u^{2}}{u^{2}}+\left(\frac{u}{R_{\mathrm{adS}}}\right)^{2} \frac{g_{5+1}}{R_{\mathrm{adS}}^{2}}\right],
$$

with now $u^{2}=2 R_{\mathrm{adS}} r$.

\subsection{String Branes}

The near-horizon geometries of $p$-branes in type II string theory are not of the form

$$
\operatorname{adS}_{p+2} \times S^{D-p-2},
$$

with the exception of the D3-brane of type IIB string theory, for which the near-horizon geometry is $\operatorname{adS}_{5} \times S^{5}$. For other $\mathrm{D} p$-branes $(p \neq 3)$ the nearhorizon geometry is conformal to (9), the conformal factor being nontrivial, and either singular for $p<3$ or zero for $p>3$ as $r \rightarrow 0$.

Geometries of the form

$$
\operatorname{adS}_{p+2} \times S^{d} \times M_{D-p-2}
$$

for some space $M$ also arise, and compactifying on $M$ leads to a adS $\mathrm{S}_{p+2} \times S^{d}$ geometry. For example, $\operatorname{adS}_{3} \times S^{3}$ arises from a D1-brane lying inside a D5-brane [59], while $\operatorname{adS}_{2} \times S^{2}$ is the near-horizon geometry for the extreme Reissner-Nordström black hole.

\subsubsection{The D3-brane in Type IIB}

The metric for the D3-brane of type IIB is given by [50],

$$
g=H^{-\frac{1}{2}} g_{3+1}+H^{\frac{1}{2}} g_{6}
$$

where $g_{3+1}$ is the metric on the Minkowski worldvolume of the brane $\mathbb{E}^{3,1}$ and $g_{6}$ is the euclidean metric on the transverse $\mathbb{E}^{6}$. The self-dual 5 -form 
$F$ has (quantised) flux on the unit transverse five-sphere $S^{5} \subset \mathbb{E}^{6}$ and the dilaton is constant. $H$ is again harmonic in $\mathbb{E}^{6}$ and the unique spherically symmetric solution with $\lim _{r \rightarrow \infty} H(r)=1$ is

$$
H(r)=1+\frac{a^{4}}{r^{4}} ; \quad a^{4} \equiv 4 \pi g N \ell_{s}^{4}
$$

where $g$ is the string coupling constant, given by the exponential of the constant dilaton, and $\ell_{s}=\sqrt{\alpha^{\prime}}$ is the string length. The solution corresponds to $N$ parallel D3-branes at $r=0$. The ten-dimensional Planck length is $\ell_{p} \equiv g^{\frac{1}{4}} \ell_{s}$, so that $a$ can be rewritten as

$$
a^{4}=4 \pi N \ell_{p}^{4}
$$

The near-horizon analysis is similar to that for the M2 and M5 branes above, and the near-horizon geometry is

$$
\operatorname{adS}_{5} \times S^{5},
$$

where the anti-de Sitter and sphere radii are now equal, $R_{\mathrm{adS}}=a=(4 \pi N)^{\frac{1}{4}} \ell_{p}$.

\section{Branes at Conical Singularities}

All the branes considered in the previous section interpolate between flat Minkowski spacetime asymptotically far away from the brane and ad $\mathrm{S}_{p+2} \times S^{d}$ near the brane horizon. Both of these vacua are maximally supersymmetric and the branes themselves preserve $\nu=\frac{1}{2}$ of the supersymmetry. In this section we discuss the brane solutions of [23] that interpolate between nearhorizon geometries of the form adS $\mathrm{an}_{p+2} \times X$ and an asymptotic geometry of the form $M_{p+1} \times C(X)$, where $X$ is a $d$-dimensional Einstein manifold and $C(X)$ is the cone over $X$. This can be interpreted as a set of coincident branes at a conical singularity in a Ricci-flat manifold. These brane solutions interpolate between vacua which are not maximally supersymmetric and the brane solutions themselves will preserve a smaller fraction $\nu<\frac{1}{2}$ of the supersymmetry. In this section we will characterise those branes with $\nu \neq 0$ in terms of the holonomy group of the transverse space.

\subsection{Cone Branes}

The $p$-brane solutions above all have metrics of the form

$$
g=H^{-\alpha} g_{p+1}+H^{\frac{2}{\beta}} g_{E}
$$


where

$$
H(r)=1+\left(\frac{a}{r}\right)^{\beta}
$$

$g_{p+1}$ is the Minkowski metric on the worldvolume of the $p$-brane, and where $g_{E}$ is the flat Euclidean metric on $\mathbb{E}^{D-p-1}$. For the M2-brane, M5-brane, and D3-brane, $\beta=6,3,4$ respectively, and $\alpha=1-\frac{2}{\beta}$. The Euclidean metric $g_{E}$ can be written as

$$
g_{E}=d r^{2}+r^{2} g_{S}
$$

where $g_{S}$ is the round metric on the unit sphere $S^{d} \subset \mathbb{E}^{d}$ and $r$ is a radial coordinate. In the near-horizon limit in which the constant term in $H$ can be dropped, the metric (13) becomes

$$
g \sim\left(\frac{r}{a}\right)^{\alpha \beta} g_{p+1}+a^{2} r^{-2} d r^{2}+a^{2} g_{S},
$$

which is the metric on $\operatorname{adS}_{p+2} \times S^{d}$, as can be seen by changing variables to $u=R_{\mathrm{adS}}\left(\frac{r}{a}\right)^{\frac{\alpha \beta}{2}}$, where the anti-de Sitter radius is $R_{\mathrm{adS}}=\frac{2 a}{\alpha \beta}$.

Replacing the sphere $S^{d}$ by any other $d$-dimensional Einstein manifold $X_{d}$ with the same cosmological constant $\Lambda=d-1$ gives another solution of the field equations on $\operatorname{adS}_{p+2} \times X_{d}$, and we will be interested in those choices of $X$ that give spontaneous compactifications to anti-de Sitter space that preserve some nonzero fraction $\nu$ of the supersymmetry. Note that as $X$ is a complete Einstein space with positive cosmological constant, it follows by Myer's Theorem that it is compact (see, e.g., [18]). There is a brane solution of the form [23]

$$
g=H^{-\alpha} g_{p+1}+H^{\frac{2}{\beta}} g_{C},
$$

where $H(r)$ is again given by (14) and the metric $g_{C}$ is given by replacing the spherical metric $g_{S}$ with the metric of $X_{d}$ in (15) to give

$$
g_{C}=d r^{2}+r^{2} g_{X} \text {. }
$$

The transverse space to the brane is now the metric cone $C \equiv C(X)$ over $X$ with topology $C \cong \mathbb{R}^{+} \times X$, with $\mathbb{R}^{+}$the open half-line $0<r<\infty$ and metric $g_{C}$. Since $X$ is Einstein with $\Lambda=\operatorname{dim} X-1$, it follows that $C$ is Ricci-flat; however unlike the case of the sphere, where $C$ is actually flat, $C$ is now metrically singular at the apex of the cone $r=0$.

In the near-horizon region $r \ll a$, the constant term in $H$ can be dropped and the metric becomes approximately

$$
g \sim\left(\frac{a}{r}\right)^{\alpha \beta} g_{p+1}+a^{2} r^{-2} d r^{2}+a^{2} g_{C},
$$


which is the metric on $\operatorname{adS}_{p+2} \times X_{d}$. For large $r, H \sim 1$ and the metric becomes

$$
g \sim g_{p+1}+g_{C},
$$

on the product $M_{p+1} \times C(X)$ of $(p+1)$-dimensional Minkowski space with the cone $C(X)$. We interpret these solutions as describing coincident branes located at the conical singularity of $C(X)$, or cone branes for short. Note that whereas the solution $M_{p+1} \times C(X)$ has a conical singularity at $r=0$, the brane metric (16) approaches the metric of $\operatorname{adS}_{p+2} \times X_{d}$ as $r \rightarrow 0$, which is non-singular at $r=0$. However $r=0$ is a horizon for the brane and the solution can be continued through the horizon. In general there will be a singularity inside the horizon.

These solutions can in principle be generalised by replacing $H$ with more general harmonic functions on the cone $C(X)$.

\subsection{Supersymmetry and Holonomy}

In this subsection we wish to describe the amount of supersymmetry preserved by the solutions adS $\mathrm{S}_{p+2} \times X_{d}$ and $M_{p+1} \times C(X)$, and the brane solution (16) that interpolates between them. In each case, the number of supersymmetries is the number of linearly independent spinors $\epsilon$ such that the supersymmetry variation of the gravitini vanish in this background: $\delta_{\epsilon} \Psi=0$. On adS $S_{p+2} \times X_{d}$, such spinors are of the form $\chi \otimes \psi$ where $\chi$ is a Killing spinor on $\operatorname{adS}_{p+2}$ and $\psi$ is a Killing spinor on $X$, satisfying

$$
\nabla_{M}^{(X)} \psi=\epsilon \frac{1}{2} \Gamma_{M} \psi
$$

where $\epsilon$ is either +1 or -1 , depending on the field $F$. For simplicity, and also for ease of comparison with the mathematical literature on Killing spinors, we have rescaled the metric on the Einstein manifold in such a way that the coefficient on the right-hand side of equation (17) is $\epsilon \frac{1}{2}$. The number of supersymmetries of the $\operatorname{adS}_{p+2} \times X_{d}$ solution is given by $\mathcal{N} n_{A} n_{X}^{ \pm}$where $n_{X}^{ \pm}$is the number of Killing spinors on $X$ with $\epsilon= \pm 1, n_{A}=2^{\left\lfloor\frac{\left.\frac{p+2}{2}\right\rfloor}{2}\right.}$ is the number of Killing spinors on adS $\mathrm{S}_{p+2}$ and $\mathcal{N}=1$ for $M$ theory and $\mathcal{N}=2$ for Type II strings.

If the dimension of $X$ is even, then there are as many solutions with $\epsilon=1$ as with $\epsilon=-1$, whereas if $\operatorname{dim} X$ is odd, then changing the sign of $\epsilon$ corresponds to reversing the orientation of $X$. Thus for odd-dimensional $X$, the number of solutions depends on the orientation. For example, for $d=7$, if there are $n_{X}>0$ Killing spinors with one choice of orientation of $X$, there 
will be no Killing spinors with the opposite choice of orientation, unless $X$ is the round 7-sphere, in which case $n_{X}=8$ with either choice of orientation [24]. We will see below that this is very easy to understand in terms of the holonomy of the cone $C(X)$.

On $M_{p+1} \times C(X)$, the supersymmetries are of the form $\chi \otimes \psi$ where $\chi$ and $\psi$ are covariantly constant spinors on Minkowski space $M_{p+1}$ and the cone $C(X)$, respectively. In particular, $\psi$ satisfies

$$
\nabla^{(C)} \psi=0 \text {. }
$$

The number of supersymmetries of the $M_{p+1} \times C(X)$ solution is then given by $\mathcal{N} n_{M} n_{C}$ where $n_{C}$ is the number of covariantly constant spinors on the cone $C(X)$ and $n_{M}=2^{\left\lfloor\frac{p+1}{2}\right\rfloor}$ is the number of parallel spinors on $M_{p+1}$.

As shown in [4], there is a one to one correspondence between Killing spinors on $X$ satisfying (17) and covariantly constant spinors on $C(X)$ satisfying (18). Each covariantly constant spinor on $C(X)$ restricts to a Killing spinor on $X$ satisfying (17) for a particular orientation $\epsilon$ of $X$. If $\operatorname{dim} X$ is odd, so that $\operatorname{dim} C(X)$ is even, then the sign of $\epsilon$ is also correlated to the chirality of the parallel spinor on $C$. If $d \equiv \operatorname{dim} X$ is even, so that $\operatorname{dim} C(X)$ is odd, then the sign of $\epsilon$ depends on which one of the two irreducible representations of the Clifford algebra $\mathrm{C} \ell_{d+1}$ we use to embed the unique spinor representation of $\operatorname{Spin}_{d+1}$.

In the round 7-sphere compactification of $\mathrm{M}$ theory, there are 8 solutions of (17) with $\epsilon=1$ and 8 solutions with $\epsilon=-1$, of which only 8 have the right sign to be Killing spinors, while $C\left(S^{7}\right)=\mathbb{E}^{8}$ has 16 covariantly constant spinors. In this case, $n_{X}^{ \pm}=8, n_{C}=16, n_{A}=4$ and $n_{M}=2$, so there are 32 supersymmetries on both $M \times C=\mathbb{E}^{10,1}$ and $\operatorname{adS}_{4} \times S^{7}$. For other $X_{7}$, there are only solutions of (17) with one particular orientation $\epsilon$, and parallel spinors on $C(X)$ restrict to spinors satisfying (17) with that choice of orientation. This is because the spinors left invariant by any of the possible holonomy groups which act irreducibly in eight dimensions all have the same chirality. On the other hand, as will be seen shortly, when $X$ is an Einstein manifold admitting Killing spinors and has dimension $4 k+1$, both orientations give the same numbers of supersymmetries. For Type IIB compactifications on 5-manifolds, this conflicts with a claim made in [22]. At any rate, we are interested in the supersymmetric cases, so we choose $\epsilon=+1$.

For M2-, D3- and M5-branes, $n_{A}=4,4,8$ respectively, whereas $n_{M}=$ $2,4,8$. Using our result for the numbers of asymptotic and near-horizon supersymmetries we find that for M2- and D3-branes the number of supersymmetries of the near-horizon geometry $\operatorname{adS}_{p+2} \times X_{d}$ is twice the number of 
supersymmetries for the asymptotic conical geometry $M_{p+1} \times C(X)$, unless $X$ is a round sphere, in which case the number of supersymmetries is the same in both cases. Applying this to the M5-brane gives the same number of supersymmetries asymptotically and near the horizon, but in this case, as we shall see, the only smooth spaces $X$ that admit Killing spinors are $S^{4}$ and $\mathbb{R} P^{4}$. In each of these cases, the number of supersymmetries can be further reduced by orbifolding. In particular, for the M5-brane with nonspherical near-horizon geometry, the asymptotic space is actually an orbifold $M_{6} \times \mathbb{R}^{5} / \Gamma$ and in this case the near-horizon limit has twice the number of supersymmetries as the asymptotic region.

As an example, consider the squashed 7-sphere of [23], which has $n_{X}^{+}=1$, and $n_{X}^{-}=0$, so that $\nu=\frac{1}{8}$ or $\nu=0$ for the squashed 7 -sphere compactification, depending on the orientation. The cone has one parallel spinor, so the near-horizon geometry has $\nu=\frac{1}{8}$ or $\nu=0$, while the asymptotic conical geometry has $\nu=\frac{1}{16}$.

In summary, the amount of supersymmetry on the brane and in the nearhorizon geometry is determined by the number of parallel spinors on the cone $C(X)$, and this can now be analysed group-theoretically.

Assume that the base of the cone, $X$, is simply-connected. Its cone $C$ will be simply-connected also, since $X$ and $C$ are homotopy equivalent. Simply-connected manifolds admitting parallel spinors are classified by their holonomy group [70]. Because $C$ is Ricci-flat, we know that it cannot be a locally symmetric space. Moreover, by a theorem of Gallot [33], the holonomy group acts on $C$ irreducibly unless $C$ is flat, in which case $X$ is the round sphere. Therefore, we need only consider irreducible holonomy groups of manifolds which are not locally symmetric. In other words, those in Berger's table (see, e.g., [7, 66]).

Of those, the ones which admit parallel spinors are given in the following table, which also lists the number $n$ (or $\left(n_{L}, n_{R}\right)$ in even dimension) of linearly independent parallel spinors.

If $X$, and hence $C$, were not simply-connected, then we could still use part of the above analysis. Gallot's theorem still applies and the existence of parallel spinors constrains the restricted holonomy group of the manifold to be contained in the above Table. However a spinor which is invariant under the restricted holonomy group need not be parallel, because it may still transform nontrivially under parallel transport along noncontractible loops. Therefore the number of parallel spinors in $C$ will be at most the number shown in Table 1. 


\begin{tabular}{|c|c|c|c|}
\hline $\operatorname{dim}$ & Holonomy & Geometry & $n$ \\
\hline \hline $4 k+2$ & $\mathrm{SU}_{2 k+1}$ & Calabi-Yau & $(1,1)$ \\
$4 k$ & $\mathrm{SU}_{2 k}$ & Calabi-Yau & $(2,0)$ \\
$4 k$ & $\mathrm{Sp}_{k}$ & hyperkähler & $(k+1,0)$ \\
7 & $G_{2}$ & exceptional & 1 \\
8 & $\mathrm{Spin}_{7}$ & exceptional & $(1,0)$ \\
\hline
\end{tabular}

Table 1: Manifolds admitting parallel spinors

\section{Near-horizon Geometries of Cone Branes}

In this section we will discuss the relation between the geometry of $X$ and the holonomy of its cone $C$. We will do this in some generality, and give the relation between the geometry of $X$ and the number of supersymmetries preserved by a adS $\times X$ solution, when one exists, and this characterises the near-horizon geometries of the supersymmetric cone branes discussed in the previous section.

On every cone there is a privileged vector field $\xi=r \partial_{r}$, called the $E u$ ler vector, which generates the rescaling diffeomorphisms. Moreover on any manifold of reduced holonomy, the holonomy principle guarantees the existence of certain parallel tensors, corresponding to the singlets under the holonomy group in the tensor products of the holonomy representation. Therefore on a cone of reduced holonomy we will be able to build interesting geometric structures out of these parallel tensors and the Euler vector. These structures will in turn induce interesting geometric structures on $X$, which we identify with $\{1\} \times X \subset C$. These geometric structures are summarised in the following table, which also lists the numbers $\left(n_{+}, n_{-}\right)$of Killing spinors; that is, solutions of equation (17), with $n_{ \pm}$the number of solutions with $\epsilon= \pm 1$.

We now proceed to describe these geometries in detail.

\subsection{Cones with $\operatorname{Spin}_{7}$ Holonomy}

Any eight-dimensional manifold with $\mathrm{Spin}_{7}$ holonomy possesses a parallel self-dual 4-form $\Omega$, known as the Cayley form. Contracting the Euler vector $\xi$ into the Cayley form gives a 3 -form on $C$ which restricts to a 3 -form $\phi$ on 


\begin{tabular}{|c|c|c|c|}
\hline $\operatorname{dim} X$ & Holonomy of $C$ & Geometry of $X$ & $\left(n_{+}, n_{-}\right)$ \\
\hline \hline$d$ & $\{1\}$ & round sphere & $\left(2^{\lfloor d / 2\rfloor}, 2^{\lfloor d / 2\rfloor}\right)$ \\
$4 k-1$ & $\mathrm{Sp}_{k}$ & 3-Sasaki & $(k+1,0)$ \\
$4 k-1$ & $\mathrm{SU}_{2 k}$ & Sasaki-Einstein & $(2,0)$ \\
$4 k+1$ & $\mathrm{SU}_{2 k+1}$ & Sasaki-Einstein & $(1,1)$ \\
6 & $G_{2}$ & nearly Kähler & $(1,1)$ \\
7 & $\mathrm{Spin}_{7}$ & weak $G_{2}$ holonomy & $(1,0)$ \\
\hline
\end{tabular}

Table 2: Manifolds admitting real Killing spinors

$X:$

$$
\phi(u, v, w) \equiv \Omega(\xi, u, v, w),
$$

for any vectors $u, v, w$ tangent to $X$. In fact, one can write the Cayley form restricted to $X \subset C$ as

$$
\Omega=d r \wedge \phi+\star \phi,
$$

with $\star$ the Hodge operator on $X$. Notice that $d r$ is a 1 -form on $C$, and by its restriction to $X$ we simply mean evaluating it at $X \subset C$ on vector fields tangent to $C$. In other words, $d r$ acts both on vectors tangent and normal to $X$. From the fact that $\Omega$ is parallel in $C$, it follows that, in $X, \phi$ obeys

$$
\nabla \phi=\star \phi .
$$

This condition says that $X$ has weak $G_{2}$ holonomy [39], and we say that $\phi$ defines a nearly parallel $G_{2}$ structure. In fact, as proven in [4] a manifold has weak $G_{2}$ holonomy if and only if its metric cone has holonomy contained in $\mathrm{Spin}_{7}$. In the early Kaluza-Klein literature it would have been said that $X$ has $G_{2}$ Weyl holonomy, but we will not follow this nomenclature.

\subsection{Cones with $G_{2}$ holonomy}

On any seven-dimensional manifold with $G_{2}$ holonomy there is a parallel 3form $\Phi$, known as the associative form. Contracting the Euler vector into the associative form yields a 2 -form on $C$ which restricts to a 2 -form $\omega$ on $X$ :

$$
\omega(u, v) \equiv \Phi(\xi, u, v)
$$


for any vectors $u, v$ tangent to $X$. We can define a $(1,1)$-tensor $J$ on $X$ as follows:

$$
\langle J(u), v\rangle \equiv \omega(u, v) .
$$

It is possible to show [4] that $J$ is an orthogonal almost complex structure:

$$
J^{2}=-\mathbb{1} \quad \text { and } \quad\langle J(u), J(v)\rangle=\langle u, v\rangle,
$$

whence $X$ is an almost hermitian manifold. From the fact that $\Phi$ is parallel on $C$, it follows that on $X$,

$$
\nabla_{v} J(v)=0 \quad \text { for any } v
$$

although $\nabla J \neq 0$. Moreover $J$ is not integrable. This means that $X$ is a non-Kähler nearly Kähler manifold [38].

In fact, the converse is also true and a six-dimensional manifold is nonKähler nearly Kähler if and only if its cone has holonomy contained in $G_{2}$ [4]. A different proof that a six-dimensional manifold admits Killing spinors if and only if it is non-Kähler nearly Kähler appeared earlier in [44].

Nearly Kähler 6-manifolds share many properties with Calabi-Yau 3folds. For example, there is a natural 3 -form defined by contracting the Euler vector $\xi$ with the coassociative 4 -form $\star \Phi$ on the cone. In particular, nearly Kähler 6-manifolds have vanishing first Chern class [41]. For example, $S^{6} \cong G_{2} / \mathrm{SU}_{3}$ is nearly Kähler, but cannot be Kähler because $H^{2}\left(S^{6}\right)$ is trivial.

\subsubsection{Scholium on Almost Hermitian Manifolds}

The notion of nearly Kähler manifolds should not be confused with the notion of an almost Kähler manifold, which simply means an almost hermitian manifold whose associated 2 -form $\omega$ is closed; or in other words, a symplectic manifold with a compatible almost complex structure. Unfortunately, given the many different generalisations of the notion of Kähler manifolds, there is a large possibility for confusion, so we digress momentarily to settle the notation once and for all. As shown in [42], there are 16 classes of almost hermitian manifolds, the class of Kähler manifolds being but one of them. The classes are defined in the following way.

One can define almost hermitian geometry in terms of $G$-structures. Just like a Riemannian metric $g$ on an $2 n$-dimensional manifold $X$ allows us to reduce the structure group of the tangent bundle from $\mathrm{GL}_{2 n} \mathbb{R}$ to $\mathrm{O}_{2 n}$, an 
almost hermitian structure $(g, J)$ allows us to reduce the group further to $\mathrm{U}_{n}$. This means that tensor bundles can be consistently decomposed into $\mathrm{U}_{n}$-irreducible sub-bundles. Let $\omega$ be the associated 2-form. Its covariant derivative $\nabla \omega$ is a section through a sub-bundle $W \subset T^{*} \otimes \bigwedge^{2} T^{*}$, with $T^{*}$ the cotangent bundle of $X . W$ is not irreducible under $\mathrm{U}_{n}$, but rather decomposes into four irreducible components $W=\bigoplus_{i=1}^{4} W_{i}$. There are therefore sixteen $\mathrm{U}_{n}$-invariant sub-bundles (not necessarily irreducible) in $W:\{0\}, W_{i}, W_{i} \oplus W_{j}(i \neq j), \ldots, W$. The sixteen classes of hermitian manifolds correspond to these sixteen sub-bundles: a manifold $X$ belonging to the class corresponding to the sub-bundle of $W$ to which $\nabla \omega$ belongs.

Clearly $\nabla \omega=0$ corresponds to the class of Kähler manifolds; but there are other classes of manifolds which are close to Kähler in some sense. The class of non-Kähler nearly Kähler manifolds can be shown to be the one for which $\nabla \omega=\frac{1}{3} d \omega \neq 0$. The class of almost Kähler manifolds consists of those for which $d \omega=0$. To make matters even more confusing there exist also semi-Kähler manifolds for which $d \star \omega=0$ and quasi-Kähler manifolds which have a more complicated definition which we will not need. For details the reader is referred to [42].

\subsection{Calabi-Yau Cones}

The class of Calabi-Yau $n$-folds is the class of compact Ricci-flat Kähler $n$-folds. Let us concentrate first on the Kähler condition. Any Kähler manifold has a parallel 2-form, the Kähler form, and a corresponding orthogonal complex structure $I$ which is also parallel. Together with the Euler vector, we can therefore build two objects: a 1-form obtained by contracting the Euler vector into the Kähler form, and a vector obtained by acting with the complex structure on the Euler vector. The vector is clearly tangent to $X$ and restricts there to a vector $\chi$, whereas the 1 -form restricts to a 1 -form $\theta$ on $X$ which is naturally dual to $\chi$ :

$$
\chi=I(\xi) \quad \text { and } \quad \theta=\langle\chi,-\rangle .
$$

It follows from the definition that $\chi$ has unit norm and is a Killing vector. Furthermore, the covariant derivative of $\chi$ defines a $(1,1)$-tensor $T \equiv \nabla \chi$ in $X$,

$$
T(v)=\nabla_{v} \chi,
$$

which, because $I$ is parallel in $C$, satisfies the following identity:

$$
\nabla_{u} T(v)=\theta(v) u-\langle u, v\rangle \chi
$$


The triple $(\chi, \theta, T)$ defines a Sasaki structure on $X$. More precisely, a triple $(\chi, \theta, T)$ on an odd-dimensional Riemannian manifold $X$, where $\chi$ is a unit norm Killing vector, $\theta$ its dual 1-form, and $T=\nabla \chi$ obeys equation (20), is called a Sasaki structure on $X$ [67], and $X$ is called Sasakian. Equivalently [4], a manifold $X$ is Sasakian if and only if its metric cone $C(X)$ is Kähler.

If in addition $C(X)$ is Ricci-flat (hence Calabi-Yau), then $X$ is Einstein. We say that $X$ is Sasaki-Einstein. Therefore a manifold is Sasaki-Einstein if and only if its metric cone is Calabi-Yau [29, 30,4].

This means, in particular, that a Sasaki-Einstein manifold $X$ of dimension $2 n+1$ also possesses two distinguished $n$-forms obtained by contracting the Euler vector $\xi$ into the real and imaginary parts of the complex volume $(n+1)$-form on $C(X)$.

The Killing vector $\chi$ in a $(2 n+1)$-dimensional Sasakian manifold $X$ defines a foliation whose leaves are the integral curves of $\chi$. The manifold is called regular if these curves are closed and have the same length. If this is the case, $\chi$ defines a $\mathrm{U}_{1}$ action on $X$ and $X$ can be understood as a circle bundle over the orbit space, a $2 n$-dimensional manifold $M$, which can be shown to be Kähler. Moreover if $X$ is Sasaki-Einstein then $M$ is KählerEinstein. Regularity is a very stringent condition which is not satisfied by most Sasaki(-Einstein) manifolds. When the integral curves of $\chi$ are closed but of different lengths, the orbit space is an orbifold which is everywhere smooth except at a finite number of points $[29,10]$.

\subsection{Hyperkähler Cones}

In a hyperkähler manifold we have a parallel quaternionic structure consisting of three orthogonal complex structures $I, J, K$ satisfying the quaternion algebra, as well as their corresponding Kähler forms: $\omega_{I}, \omega_{J}, \omega_{K}$. From the discussion above, on a cone $C(X)$ each complex structure gives rise to one Sasaki structure on $X$. Moreover the fact that the three complex structures on $C(X)$ satisfy the quaternion algebra means that the Killing vectors in the three Sasaki structures are orthonormal and obey an $\mathfrak{s u}_{2}$ Lie algebra. Three Sasaki structures satisfying these conditions define a 3-Sasaki structure on $X$ (see [10] for the latest word on 3-Sasakian manifolds). Equivalently, one can prove the converse and show that a manifold is 3-Sasakian if and only if its cone is hyperkähler $[30,4,11]$.

The three Killing vectors define a foliation of the 3-Sasakian manifold $X$. We say that $X$ is regular if the foliation fibres. This means that $X$ is 
an $\mathrm{SU}_{2}$ or $\mathrm{SO}_{3}$ bundle over a quaternionic Kähler space $Q$. Equivalently, $X$ is a circle bundle over the twistor space $Z$ of $Q$. If $X$ is not regular, but the Killing vectors are complete, then the orbit space defines a quaternionic Kähler orbifold [10].

\section{Examples}

In this section we list the known near-horizon geometries for different types of cone branes. The homogeneous examples are all known from the early days of Kaluza-Klein supergravity, but we do list some non-homogeneous examples as well. For each type of cone brane we discuss the possible smooth near-horizon geometries and where applicable we discuss orbifolds.

\subsection{M2 Cone Branes}

The transverse space to an $\mathrm{M} 2$ cone brane is the metric cone $C_{8}$ over a 7-dimensional manifold $X_{7}$. From Table 2 one can read off the following possibilities for simply-connected $X_{7}$, which are listed in Table 3 . In the non-simply-connected case the number of Killing spinors is at most the one shown in the table.

\begin{tabular}{|c|c|c|}
\hline Geometry of $C_{8}$ & Geometry of $X_{7}$ & $\left(n_{+}, n_{-}\right)$ \\
\hline \hline flat & round $S^{7}$ & $(8,8)$ \\
hyperkähler & 3-Sasaki & $(3,0)$ \\
Calabi-Yau & Sasaki-Einstein & $(2,0)$ \\
Spin $_{7}$ holonomy & weak $G_{2}$ holonomy & $(1,0)$ \\
\hline
\end{tabular}

Table 3: Seven-dimensional near-horizon geometries

Besides the sphere and its quotients, we have the following types of nearhorizon geometries.

\subsection{1 $X_{7}$ is 3 -Sasakian}

We must distinguish between regular and non-regular 3-Sasakian 7-manifolds. The regular manifolds were classified in $[30,11]$ and they all happen to be homogeneous spaces. Therefore they have been known since the early days 
of Kaluza-Klein supergravity [17]. In the present context they have been discussed in [14]. Apart from $\mathrm{Sp}_{2} / \mathrm{Sp}_{1} \cong S^{7}$ and $\mathrm{Sp}_{2} /\left(\mathrm{Sp}_{1} \times \mathbb{Z}_{2}\right) \cong \mathbb{R} \mathbb{P}^{7}$, the only other homogeneous regular example is $\left(\mathrm{SU}_{3} \times \mathrm{U}_{1}\right) /\left(\mathrm{U}_{1} \times \mathrm{U}_{1}\right)$, which is called $N^{010}$ in [14] and $N(1,1)$ in [24].

On the other hand there are an infinite number of different non-regular 3-Sasakian 7-manifolds. The topology of a seven-dimensional 3-Sasakian manifold $X_{7}$ is highly constrained. First of all, Bochner's theorem implies that the first Betti number of a 3-Sasakian manifold vanishes: $b_{1}=0$. In seven dimensions, $b_{6}=0$ by Poincaré duality. Furthermore, as shown in $[32,31], b_{3}=b_{4}=0$, so that the only nonzero Betti numbers are $b_{2}=$ $b_{5}$. Recently an infinite family with arbitrary $b_{2}$ has been constructed in $[13,9]$. This implies that there exist 3 -Sasakian 7 -manifolds of every possible rational homotopy type allowed. These examples are toric; that is, they admit an action of a torus $\mathbb{T}^{2}$ preserving the 3-Sasakian structure. They are constructed via a 3-Sasakian quotient [12] akin to the hyperkähler quotient [49]. Indeed, the two quotients are related via the cone construction. In other words, suppose $X_{7}$ is a 3-Sasakian manifold and $C_{8}$ is its hyperkähler cone. Then if $C_{8}$ admits a triholomorphic action commuting with the Euler vector, then the hyperkähler quotient of $C_{8}$ is a cone over the 3-Sasaki quotient of $X_{7}$. The cones over the toric 3 -Sasakian manifolds in $[13,9]$ form a subclass of the toric hyperkähler manifolds studied in [8] and which have been considered in the context of intersecting branes in [34].

All 3-Sasakian manifolds have an infinitesimal $\mathfrak{s u}_{2} \cong \mathfrak{s o}_{3}$ isometry. If the Killing vectors are complete, they integrate to an action of $\mathrm{SU}_{2}$ or $\mathrm{SO}_{3}$. In the regular case, the orbit space is smooth; otherwise not. In any case, the infinitesimal isometry is a generic feature of these manifolds and one which will play a role in Section 6, when we discuss the dual field theories.

\subsection{2 $X_{7}$ is Sasaki-Einstein}

There are many known Sasaki-Einstein 7-manifolds. The regular examples can be understood as $U_{1}$ bundles over Kähler-Einstein 3-folds [64]. They have not been classified. The known results are summarised in the following table [31].

The first four examples are homogeneous and have therefore appeared in the early Kaluza-Klein literature. The first three are listed using the nomenclature of [17]. In [24, 31] these spaces are called $N(1,1), Q(1,1,1)$ and $M(3,2)$, respectively. Here $V(2 \mid 5)$ is the Stiefel manifold of orthonormal oriented 2-frames in $\mathbb{R}^{5}$ and $G(2 \mid 5)$ is the Grassmannian of oriented 2-planes 


\begin{tabular}{|c|c|}
\hline$K_{6}$ & $X_{7}$ \\
\hline \hline$F(1,2 \mid 3)$ & $N^{010} \cong\left(\mathrm{SU}_{3} \times \mathrm{SU}_{2}\right) /\left(\mathrm{SU}_{2} \times \mathrm{U}_{1}\right)$ \\
$\mathbb{C P}^{1} \times \mathbb{C P}^{1} \times \mathbb{C P}^{1}$ & $Q^{111} \cong\left(\left(\mathrm{SU}_{2}\right)^{3} \times \mathrm{U}_{1}\right) /\left(\left(\mathrm{U}_{1}\right)^{3} \times \mathrm{U}_{1}\right)$ \\
$\mathbb{C P}^{2} \times \mathbb{C P}^{1}$ & $M^{110} \cong\left(\mathrm{SU}_{3} \times \mathrm{SU}_{2} \times \mathrm{U}_{1}\right) /\left(\mathrm{SU}_{2} \times \mathrm{U}_{1} \times \mathrm{U}_{1}\right)$ \\
$G(2 \mid 5)$ & $V(2 \mid 5) \cong\left(\mathrm{SO}_{5} \times \mathrm{U}_{1}\right) /\left(\mathrm{SO}_{3} \times \mathrm{U}_{1}\right)$ \\
$P_{k} \times \mathbb{C P}$ & $M_{k}^{7}(3 \leq k \leq 8)$ \\
\hline
\end{tabular}

Table 4: Known regular Sasaki-Einstein 7-manifolds $X_{7}$, as $\mathrm{U}_{1}$ bundles $X_{7} \rightarrow$ $K_{6}$ over Kähler-Einstein 3 -folds

in $\mathbb{R}^{5} . F(1,2 \mid 3)$ is the complex flag manifold in $\mathbb{C}^{3}$ consisting of pairs $(\ell, \pi)$ where $\pi$ is a complex plane in $\mathbb{C}^{3}$ and $\ell \subset \pi$ is a complex line. The last class of examples does not consist of homogeneous manifolds: $P_{k}$ are the del Pezzo surfaces consisting of blowing up $k$ points in general position on $\mathbb{C P}^{2}$.

In addition, there are two infinite families of homogeneous non-regular Sasaki-Einstein 7-manifolds: the $M^{p p r}$ of $[73,15]$ and the $Q^{p p p}$ of [20]. Of course they are mentioned in [17].

\subsection{3 $X_{7}$ has Weak $G_{2}$ Holonomy}

The canonical example of a weak $G_{2}$ holonomy manifold which is not SasakiEinstein is the squashed 7-sphere, which is a homogenous space $\left(\mathrm{Sp}_{2} \times \mathrm{Sp}_{1}\right)$ $/\left(\mathrm{Sp}_{1} \times \mathrm{Sp}_{1}\right)$. It turns out that this generalises, and every 3-Sasakian 7manifold can be squashed to a manifold with weak $G_{2}$ holonomy [32, 31]. The squashing is done as follows. A 3-Sasakian manifold $X_{7}$ is foliated by the action of the Sasakian Killing vectors. At any point $p \in X_{7}$, the Killing vectors are tangent to the unique leaf $\mathcal{F}_{p}$ of the foliation passing through $p$. The tangent space to $X$ at $p$ has an orthogonal decomposition $T_{p} X=$ $\mathcal{F}_{p} \oplus\left(\mathcal{F}_{p}\right)^{\perp}$. The squashing of the metric is done by introducing a parameter $t$ and rescaling the metric on the leaves of the foliation by $t$. We can then compute the Ricci tensor as a function of $t$ and notice that there are two values for which it is Einstein: one is the original 3-Sasakian metric, and the other gives rise to a weak $G_{2}$ holonomy structure.

By squashing the infinite toric family of $[13,9]$ in this way, one obtains a huge number of weak $G_{2}$ holonomy manifolds which are not Sasaki-Einstein. These spaces are not homogeneous and hence have not been considered before in the Kaluza-Klein supergravity context. There are examples with arbitrary 
$b_{2}=b_{5}$ and all other Betti numbers vanishing, since squashing does not change the topology.

Another infinite dimensional family of weak $G_{2}$ holonomy manifolds consists of the Aloff-Wallach spaces [3] $N(k, l) \cong \mathrm{SU}_{3} / \mathrm{U}_{1}$, with $\mathrm{U}_{1} \subset \mathrm{SU}_{3}$ defined by

$$
e^{i \theta} \mapsto\left(\begin{array}{lll}
e^{i k \theta} & & \\
& e^{i \ell \theta} & \\
& & e^{-i(k+\ell) \theta}
\end{array}\right)
$$

This family is remarkable because it contains an infinite number of distinct homotopy types and even exotic pairs, i.e., homeomorphic non-diffeomorphic pairs [57]. For $k \neq 1$ or $\ell \neq 1$ these spaces admit metrics with weak $G_{2}$ holonomy. The Aloff-Wallach spaces $N(k, \ell)$ agree, allowing for some redundancy in the notation, with the $N^{p q r}$ spaces of [16]. Finally, the other weak $G_{2}$ holonomy manifold from the early Kaluza-Klein literature is the homogeneous space $\mathrm{SO}_{5} / \mathrm{SO}_{3}^{\max }$ of [17].

In summary, all known examples are homogeneous, hence previously known in the Kaluza-Klein context, except for the squashed toric 3-Sasakian manifolds.

\subsection{M5 Cone Branes}

The transverse space to an M5 cone brane is a five-dimensional cone $C_{5}$ over a four-dimensional manifold $X_{4}$. As proven in [28], any complete connected $n$-dimensional spin manifold, with $n \leq 4$, admitting real Killing spinors is locally isometric to the round $n$-sphere. Therefore the only possible smooth near-horizon geometry for an M5 cone brane is either spherical $S^{4}$ or elliptical $\mathbb{R P}^{4}$; these describe the near-horizon geometry of M5 branes at a point in $\mathbb{R}^{5}$ or on the orientifold $\mathbb{R}^{5} / \mathbb{Z}_{2}$. More generally we can consider orbifolds of $\mathbb{R}^{5} \cong \mathbb{R} \times \mathbb{C}^{2}$ which are of the form $\mathbb{R} \times \mathbb{C}^{2} / \Gamma$. Such an orbifold will preserve supersymmetry if $\Gamma$ is an ADE subgroup of the hyperkähler $\mathrm{SU}_{2}$ which acts on $\mathbb{C}^{2}$. This clearly induces a near-horizon geometry of the form $S^{4} / \Gamma$ which has two singular points induced from the orbifold fixed points of $\Gamma$ acting on $\mathbb{R} \times \mathbb{C}^{2}$. These orbifolds preserve half of the supersymmetries obtained in the maximally supersymmetric case. 


\subsection{D3 Cone Branes}

The transverse space to a D3 cone brane is a six-dimensional cone $C_{6}$ over a five-dimensional manifold $X_{5}$. From Table 2 we see that there are two simplyconnected possibilities. This means $X_{5}$ is a sphere or a Sasaki-Einstein manifold. Non-simply connected examples, e.g., $\mathbb{R P}^{5}$ or more generally $S^{5} / \Gamma$, can be obtained by taking quotients. There are an infinite number of smooth quotients $S^{5} / \Gamma$ of the sphere which possess Killing spinors. These can be determined as follows [69].

In his solution of the spherical space problem, Wolf classified all the discrete subgroups $\Gamma \subset \mathrm{SO}_{6}$, for which $S^{5} / \Gamma$ is smooth [74]. Given one such subgroup, the spin structures on $S^{5} / \Gamma$ are in one-to-one correspondence with the lifts of $\Gamma$ to an isomorphic subgroup $\tilde{\Gamma} \subset \operatorname{Spin}_{6} \cong \mathrm{SU}_{4}$; that is, to a $\tilde{\Gamma} \subset \operatorname{Spin}_{6}$ which is mapped to $\Gamma$ isomorphically under the covering map $\mathrm{Spin}_{6} \rightarrow \mathrm{SO}_{6}$. Finally, for $S^{5} / \Gamma$ with spin structure given by $\tilde{\Gamma}$, the Killing spinors in $S^{5} / \Gamma$ are precisely the $\tilde{\Gamma}$-invariant spinors in $S^{5}$. The results are as follows.

There are two families of subgroups of $\mathrm{SO}_{6}$ for which $S^{5} / \Gamma$ is smooth: $\Gamma(n, a, b)$ and $\Gamma(m, r ; n, s)$ described below.

Let $\Gamma(n, a, b)$, where $n, a, b \in \mathbb{N}$ be the cyclic subgroup of $\mathrm{SO}_{6}$ of order $n$ generated by the following element:

$$
\left(\begin{array}{ccc}
R\left(\frac{1}{n}\right) & & \\
& R\left(\frac{a}{n}\right) & \\
& & R\left(\frac{b}{n}\right)
\end{array}\right) \quad \text { with } \quad \begin{gathered}
(a, n)=(b, n)=1 \\
\end{gathered}
$$

where $R(\theta)$ denotes the rotation matrix

$$
R(\theta)=\left(\begin{array}{rr}
\cos 2 \pi \theta & \sin 2 \pi \theta \\
-\sin 2 \pi \theta & \cos 2 \pi \theta
\end{array}\right)
$$

and $(p, q)$ denotes the greatest common divisor, so that $(p, q)=1$ means that $p$ and $q$ are coprime.

Similarly let $\Gamma(m, r ; n, s)$, with $m, n, r, s \in \mathbb{N}$ and $n \equiv 0(\bmod 3)$, denote the subgroup of $\mathrm{SO}_{6}$ generated by elements $A$ and $B$ subject to the relations: $A^{m}=B^{n}=\mathbb{1}$ and $B A B^{-1}=A^{r}$. The generators can be written as

$$
A=\left(\begin{array}{ccc}
R\left(\frac{1}{m}\right) & & \\
& R\left(\frac{r}{m}\right) & \\
& & R\left(\frac{r^{2}}{m}\right)
\end{array}\right) \quad \text { and } \quad B=\left(\begin{array}{ccc}
0 & \mathbb{1} & 0 \\
0 & 0 & \mathbb{1} \\
R\left(\frac{3 s}{n}\right) & 0 & 0
\end{array}\right)
$$


where $\mathbb{1}$ is a $2 \times 2$ identity matrix, and where $((r-1) n, m)=\left(s, \frac{n}{3}\right)=1$ and $r \not \equiv r^{3} \equiv 1(\bmod m)$ and $\mathbb{1}$ is the identity matrix of order 2 . This subgroup has order $n m$.

It is not hard to show that $S^{5} / \Gamma(n, a, b)$ has precisely one spin structure when $n$ is odd, and none for $n$ even. Similarly $S^{5} / \Gamma(m, r ; n, s)$ has precisely one spin structure when both $n$ and $m$ are odd, and none otherwise.

Computing the $\tilde{\Gamma}$-invariant spinors for each of the cases above is an easy matter. One sees that $S^{5} / \Gamma(n, a, b)$ is of type $\left(n_{+}, n_{-}\right)=(1,1)$ whenever any one of the following equalities is satisfied:

$$
a+b \pm 1=n \quad \text { or } \quad a-b= \pm 1,
$$

and has no Killing spinors otherwise. Similarly, $S^{5} / \Gamma(m, r ; n, s)$ has no Killing spinors for $n>3$; and $S^{5} / \Gamma(m, r ; 3, s), m$ odd, is of type $\left(n_{+}, n_{-}\right)=$ $(1,1)$ whenever $r^{2}+r+1 \equiv 0(\bmod m)$ and has no Killing spinors otherwise.

Notice that most of these spaces $S^{5} / \Gamma$ are not homogeneous. It is likely that, as in the case of orbifolds [54], the spectrum of the dual gauge theory associated with a near-horizon geometry of the form $\operatorname{adS}_{5} \times S^{5} / \Gamma$ agrees with the $\tilde{\Gamma}$-invariant spectrum of $\mathcal{N}=4$ supersymmetric Yang-Mills.

\begin{tabular}{|c|c|c|}
\hline Geometry of $C_{6}$ & Geometry of $X_{5}$ & $\left(n_{+}, n_{-}\right)$ \\
\hline \hline flat & round $S^{5}$ & $(4,4)$ \\
Calabi-Yau & Sasaki-Einstein & $(1,1)$ \\
\hline
\end{tabular}

Table 5: Five-dimensional near-horizon geometries

\subsection{1 $X_{5}$ is Sasaki-Einstein}

Again we distinguish between regular and non-regular Sasaki-Einstein manifolds. The regular manifolds were classified in [29]. Regularity implies that $X_{5}$ is the total space of a $\mathrm{U}_{1}$ bundle over a four-dimensional manifold $K_{4}$, which inherits a Kähler-Einstein structure. The results are summarised in Table 6 below, where as in Table 4 above, $P_{k}$ denotes the del Pezzo surfaces and $V(2 \mid 4)$ denotes the Stiefel manifold of orthonormal oriented 2-frames in $\mathbb{R}^{4}$. In the table \# denotes the operation of connected sum. For completeness the non-simply-connected cases have also been included. There are no known examples of Sasaki-Einstein 5-manifolds which are not regular. (See Problem 8.1 in [10].) 


\begin{tabular}{|c|c|}
\hline$K_{4}$ & $X_{5}$ \\
\hline \hline $\mathbb{C P}^{2}$ & $S^{5}$ \\
$\mathbb{C P}^{2}$ & $S^{5} / \mathbb{Z}_{3}$ \\
$\mathbb{C P}^{1} \times \mathbb{C P}^{1}$ & $V(2 \mid 4) \cong S^{2} \times S^{3}$ \\
$\mathbb{C P}^{1} \times \mathbb{C P}^{1}$ & $V(2 \mid 4) / \mathbb{Z}_{2}$ \\
$P_{k}, 3 \leq k \leq 8$ & $S_{k} \cong \#^{k}\left(S^{2} \times S^{3}\right)$ \\
\hline
\end{tabular}

Table 6: Regular Sasaki-Einstein 5-manifolds $X_{5}$, as $\mathrm{U}_{1}$ bundles $X_{5} \rightarrow K_{4}$ over Kähler-Einstein surfaces

The homogeneous cases were known from the early Kaluza-Klein literature. In particular, $V(2 \mid 4) \cong\left(\mathrm{SU}_{2} \times \mathrm{SU}_{2}\right) / \mathrm{U}_{1}$ is called $T^{1,1}$ in [65]. This solution $V(2 \mid 4)$ has also been discussed recently in [22] where it was denoted $Q(1,1)$ and claimed as new. These authors moreover claim that $Q(1,1)$ has type $\left(n_{+}, n_{-}\right)=(2,0)$. Since this space is simply-connected, its cone is also simply connected. If $\left(n_{+}, n_{-}\right)=(2,0)$ the cone would have to possess two covariantly constant spinors of one chirality and none of the other. Since the cone is six-dimensional the only possible holonomy group is $\mathrm{SU}_{3}$ which has two parallel spinors, one of each chirality, in contradiction with the claim in [22]. This claim seems to have propagated to [55], where the dual SCFT is claimed to have $\mathcal{N}=2$ supersymmetry. However, as pointed out correctly in [56], the theory has only $\mathcal{N}=1$ supersymmetry as expected from our geometric considerations.

\subsection{Six Dimensions}

The remaining case to consider is that of a seven-dimensional cone $C_{7}$ over a six-dimensional manifold $X_{6}$. In this case, there is no known maximally supersymmetric compactification on $S^{6}$ to an anti-de Sitter space, so the relevance of this case is unclear. From Table 2 we see that there are again two possibilities: the sphere $S^{6}$ and the nearly Kähler non-Kähler manifolds.

\begin{tabular}{|c|c|c|}
\hline Geometry of $C_{7}$ & Geometry of $X_{6}$ & $\left(n_{+}, n_{-}\right)$ \\
\hline \hline flat & round $S^{6}$ & $(8,8)$ \\
$G_{2}$ holonomy & nearly Kähler & $(1,1)$ \\
\hline
\end{tabular}

Table 7: Six-dimensional near-horizon geometries 


\subsubsection{Is Nearly Kähler}

As we mentioned above $S^{6} \cong G_{2} / \mathrm{SU}_{3}$ is nearly Kähler non-Kähler. There are other examples. First we have $\mathbb{C P}^{3}$. Although $\mathbb{C P}^{3}$ possesses a Kähler metric, the $\mathrm{SO}_{5}$-invariant one it inherits from the isomorphism $\mathbb{C P} \cong \mathrm{SO}_{5} / \mathrm{U}_{2}$ is nonKähler nearly Kähler. The same occurs for the complex flag space $F(1,2 \mid 3)$ and the $\mathrm{U}_{3}$-invariant metric coming from $F(1,2 \mid 3) \cong \mathrm{U}_{3} /\left(\mathrm{U}_{1} \times \mathrm{U}_{1} \times \mathrm{U}_{1}\right)$ [40]. The Lie group $\mathrm{Spin}_{4} \cong S^{3} \times S^{3}$ is nearly Kähler with the metric inherited from a 3-symmetric structure [44]. It cannot be Kähler because $H^{2}\left(S^{3} \times S^{3}\right)=0$. Similarly the homogeneous spaces $\mathrm{SO}_{5} /\left(\mathrm{U}_{1} \times \mathrm{SO}_{3}\right)$, $\mathrm{SO}_{6} / \mathrm{U}_{3}$ and $\mathrm{Sp}_{2} / \mathrm{U}_{2}$ with their natural homogeneous metrics are nearly Kähler non-Kähler [37].

\section{Near-Horizon Geometry And Superconformal Symmetry}

According to the conjecture in [59] superstring or $M$ theory compactified on the near-horizon geometry adS $\mathrm{S}_{p+2} \times X_{d}$ of (a large number of) parallel $p$ branes, is dual to (the 't Hooft limit of) a superconformal field theory on the worldvolume of the brane, which is the boundary of the anti-de Sitter space. The symmetry algebras of the conformal field theory and the anti-de Sitter string theory must be the same superalgebra. The anti-de Sitter symmetry algebra is the super-isometry algebra of the background; it has a bosonic subalgebra which is the isometry algebra $\mathfrak{s o}_{p+1,2} \times \mathfrak{g}$ of adS $\mathrm{S}_{p+2} \times X_{d}$, where $\mathfrak{g}$ is the isometry algebra of $X_{d}$, and there is a fermionic generator for each Killing spinor, generating the corresponding supersymmetry transformation. The same superalgebra acts as the superconformal algebra for the worldvolume theory in $M_{p+1}$, where $\mathfrak{s o}_{p+1,2}$ is the conformal algebra in $p+1$ dimensions, $\mathfrak{g}$ is a global symmetry which is the R-symmetry, or the product of the Rsymmetry with an algebra that does not act on the fermionic generators, and the fermionic generators consist of the usual supercharges in $p+1$ dimensions, together with the special supersymmetry generators.

In the dimensions of interest here, these algebras were classified by $\mathrm{Nahm}$ [62], although the case $p=3$ had been considered previously in [48]. Each such superalgebra is characterised by its bosonic subalgebra together with the representation that the supercharges are in. This means that for the anti-de Sitter vacua of interest here, the bosonic subalgebra should contain $\mathfrak{s o}_{p+1,2} \times \mathfrak{g}$, where $\mathfrak{g}$ is the isometry algebra of $X_{d}$. This information, together with Nahm's classification, is enough to identify the superconformal algebra 
of the dual theory, provided that the supercharges transform correctly. In this section we will show that the geometric structures we have listed previously are precisely enough to identify the correct superconformal algebra in all cases. We do this by identifying the generic isometries of the near-horizon geometries and by investigating how the Killing spinors transform under them.

\section{1 $d=3$ SCFTs Dual To M2-Branes}

The superconformal algebra corresponding to an $\mathcal{N}$-extended three-dimensional superconformal theory is $\mathfrak{o s p}_{4 \mid \mathcal{N}}$ which has bosonic subalgebra

$$
\mathfrak{s o}_{3,2} \times \mathfrak{s o}_{\mathcal{N}},
$$

with the supercharges transforming according to the $(\underline{4}, \underline{\mathcal{N}})$ [62]. Notice that for $\mathcal{N}=8$, there are three possible eight-dimensional representations: by $\underline{\mathbf{8}}$ we mean one of the spinorial irreducible representations.

These theories are dual to M2-branes at a conical singularity of a Ricciflat space with holonomy contained in $\operatorname{Spin}_{7}$. We should therefore identify the bosonic subalgebra as isometries of the near-horizon geometry. The $\mathfrak{s o}_{3,2}$ factor is the isometry of $\operatorname{adS}_{4}$, while the $\mathfrak{s o}_{\mathcal{N}}$ factor is a subalgebra of the isometry algebra of $X_{7}$. For a supersymmetric brane configuration, the cone can have holonomy $\{1\}, \mathrm{Sp}_{2}, \mathrm{SU}_{4}$ and $\mathrm{Spin}_{7}$, corresponding to near-horizon geometries preserving $32,12,8$ and 4 supercharges respectively. In terms of three-dimensional superconformal symmetries, each of which having four real components, these cases thus correspond to $\mathcal{N}=8,3,2,1$, respectively. We now treat each case separately.

\subsection{1 $\mathcal{N}=8$}

This is the maximally supersymmetric case, in which the near-horizon geometry is spherical. The isometry algebra of the round 7-sphere is indeed $\mathfrak{s o}_{8}$. It is clear that the Killing spinors are in the $\underline{\boldsymbol{8}}_{s}$ or $\underline{\boldsymbol{8}}_{c}$ of $\mathfrak{s o}_{8}$. Therefore one recovers, as was done originally in [59], the correct superconformal algebra.

\subsection{2 $\mathcal{N}=3$}

The corresponding near-horizon geometry in this case has a cone base which is a 3-Sasakian 7-manifold $X_{7}$. As we saw above, such a manifold possesses 
three orthonormal Killing vectors which generate an action of $\mathfrak{s u}_{2} \cong \mathfrak{s o}_{3}$. We need to check that the Killing spinors transform as the $\underline{\mathbf{3}}$ of this $\mathfrak{s o}_{3}$. This is proven in Theorem 3.1 in [60]. Alternatively, we can see this in the hyperkähler cone. Each Killing spinor in the base corresponds to a parallel spinor in the cone. From the explicit construction of the Killing vectors in the 3-Sasaki structure at the base of the cone, we see that their Lie algebra is isomorphic to the $\mathfrak{s p}_{1}$ factor in the maximal subalgebra $\mathfrak{s p}_{1} \times \mathfrak{s p}_{2} \subset \mathfrak{s o}_{8}$. Under this subalgebra, the spinor representation $\underline{\mathbf{8}}_{s}$ decomposes as

$$
\underline{\mathbf{8}}_{s} \rightarrow(\underline{\mathbf{3}}, \underline{\mathbf{1}}) \oplus(\underline{\mathbf{1}}, \underline{\mathbf{5}}) \text {. }
$$

Thus we see that the three parallel spinors transform as the $\underline{\mathbf{3}}$ of $\mathfrak{s p}_{1} \cong \mathfrak{s o}_{3}$.

\subsection{3 $\mathcal{N}=2$}

In this case the base of the cone is a Sasaki-Einstein 7-manifold $X_{7}$, and as we saw above it has a unit norm Killing spinor $\chi$. According to Theorem 2.2 in [60], the Killing spinors transform according to the real two-dimensional irreducible representation $\underline{\mathbf{2}}$ of the $\mathfrak{s o}_{2}$ action of $\chi$. Alternatively we can again work in the cone. We notice that the abelian algebra generated by $\chi$ corresponds to the $\mathfrak{u}_{1}$ factor of the maximal subalgebra $\mathfrak{u}_{1} \times \mathfrak{s u}_{4} \cong \mathfrak{u}_{4} \subset \mathfrak{s o}_{8}$. Under $\mathfrak{s o}_{8} \supset \mathfrak{u}_{1} \times \mathfrak{s u}_{4}$, the spinor representation $\underline{\mathbf{8}}_{v}$ breaks up as

$$
\underline{\mathbf{8}}_{s} \rightarrow(\underline{\mathbf{2}}, \underline{\mathbf{1}}) \oplus(\underline{\mathbf{1}}, \underline{\mathbf{6}})
$$

so that the parallel spinors do indeed transform as the $\underline{\mathbf{2}}$ of $\mathfrak{u}_{1} \cong \mathfrak{s o}_{2}$.

\subsection{4 $\mathcal{N}=1$}

This case corresponds to the cones whose base is a 7-manifold with weak $G_{2}$ holonomy. Generically the nearly parallel $G_{2}$ structure admits no infinitesimal automorphisms, although of course we gave plenty of examples above of such manifolds with a large isometry group. The Lie algebra of this group acts trivially on the Killing spinor, however. The automorphism groups of nearly parallel $G_{2}$ structures have been studied in [31]. As an example, if $X_{7}$ is the squashed 7-sphere with isometry $\mathfrak{s p}_{2} \times \mathfrak{s p}_{1}$, the symmetry algebra is $\mathfrak{o s p}_{4 \mid 1} \times \mathfrak{s p}_{2} \times \mathfrak{s p}_{1}$, with trivial R-symmetry and a symmetry $\mathfrak{s p}_{2} \times \mathfrak{s p}_{1}$ under which the supercharges are singlets. 


\section{2 $d=4$ SCFTs Dual To D3-Branes}

From the existence of supersymmetric solutions of IIB theory describing parallel D3-branes sitting at conical singularities in Ricci-flat six-dimensional cones with holonomy contained in $\mathrm{SU}_{3}$, we expect a correspondence between $d=4$ SCFTs and IIB theory in $\operatorname{adS}_{5} \times X_{5}$ with $X_{5}$ a Sasaki-Einstein 5-manifold. The superalgebras governing the superconformal field theories $[48,62]$ have bosonic subalgebras

$$
\begin{cases}\mathfrak{s o}_{4,2} \times \mathfrak{u}_{\mathcal{N}} & \text { for } \mathcal{N} \neq 4 \\ \mathfrak{s o}_{4,2} \times \mathfrak{s u}_{4} & \text { for } \mathcal{N}=4\end{cases}
$$

with the fermions in the representation $\llbracket(\underline{\underline{4}}, \underline{\mathcal{N}}) \rrbracket$, where we are using the notation $\llbracket R \rrbracket$ to mean the underlying real representation in $R \oplus R^{*}$; in other words, $\llbracket R \rrbracket \otimes_{\mathbb{R}} \mathbb{C} \cong R \oplus R^{*}$. The $\mathfrak{s o}_{4,2}$ factor is the isometry algebra of the anti-de Sitter space, and the $\mathfrak{u}_{\mathcal{N}}\left(\right.$ or $\mathfrak{s u}_{4}$ ) factor should coincide with the isometries of $X_{5}$. Moreover the isometries should act on the Killing spinors according to the above representation.

\subsection{1 $\mathcal{N}=4$}

This corresponds to the maximally supersymmetric case in four dimensions, where $X_{5}$ is the round sphere. The isometry group of the sphere has Lie algebra $\mathfrak{s o}_{6} \cong \mathfrak{s u}_{4}$, which was identified in [59] with the R-symmetry group of the $\mathcal{N}=4$ SCFT.

\subsection{2 $\mathcal{N}=2$}

This case corresponds to $X_{5} \cong S^{5} / \Gamma$ where $\Gamma$ is any of the ADE subgroups of the 'hyperkähler' $\mathrm{Sp}_{1}$ subgroup of $\mathrm{Sp}_{1} \cdot \mathrm{Sp}_{1} \cong \mathrm{SO}_{4} \subset \mathrm{SO}_{4} \times \mathrm{SO}_{2} \subset \mathrm{SO}_{6}$. The centraliser of its Lie algebra $\mathfrak{s p}_{1}$ in $\mathfrak{s o}_{6}$ is therefore $\mathfrak{s p}_{1} \times \mathfrak{s o}_{2} \cong \mathfrak{s u}_{2} \times \mathfrak{u}_{1} \cong \mathfrak{u}_{2}$, as expected. We need to verify that the action on the Killing spinors is the right one. For the sphere, all spinors are Killing, so that they transform under $\mathfrak{s o}_{6} \cong \mathfrak{s u}_{4}$ as $\llbracket \underline{4} \rrbracket$. Under the maximal $\mathfrak{s u}_{2} \times \mathfrak{s u}_{2} \times \mathfrak{s o}_{2} \subset \mathfrak{s o}_{6}$ subgroup, we have the following branchings

$$
\underline{\mathbf{4}} \rightarrow(\underline{\mathbf{1}}, \underline{\mathbf{2}})_{+1} \oplus(\underline{\mathbf{2}}, \underline{\mathbf{1}})_{-1} \quad \text { and } \quad \underline{4}^{*} \rightarrow(\underline{\mathbf{1}}, \underline{\mathbf{2}})_{-1} \oplus(\underline{\mathbf{2}}, \underline{1})_{+1} .
$$

The $\Gamma$-invariant spinors then transform according to $\llbracket \underline{\mathbf{2}}_{+1} \rrbracket$, which is precisely the $\llbracket \underline{2} \rrbracket$ of $\mathfrak{u}_{2}$. 


\subsection{3 $\mathcal{N}=1$}

In this case the cone base $X_{5}$ is a Sasaki-Einstein 5-manifold. These manifolds possess a Killing spinor which generates a $\mathfrak{u}_{1}$ subalgebra of the isometry algebra. To see how the $\mathfrak{u}_{1}$ acts on the supersymmetries it is again convenient to work in the cone. The Sasakian Killing vector generates the $\mathfrak{u}_{1}$ subalgebra of the maximal subalgebra $\mathfrak{u}_{1} \times \mathfrak{s u}_{3} \cong \mathfrak{u}_{3} \subset \mathfrak{s o}_{6}$. Under this subgroup the spinor representation $\llbracket \underline{4} \rrbracket$ of $\mathfrak{s o}_{6} \cong \mathfrak{s u}_{4}$ breaks up as

$$
\llbracket \underline{4} \rrbracket \rightarrow \llbracket \underline{\mathbf{3}}_{+1} \rrbracket \oplus \llbracket \underline{\mathbf{1}}_{-3} \rrbracket .
$$

Therefore the parallel spinors on the cone transform under $\mathfrak{u}_{1}$ as a real twodimensional representation $\llbracket(+3) \oplus(-3) \rrbracket$, in agreement with the trasnformation properties of the supercharges.

\section{3 $d=6$ SCFTs Dual To M5-Branes}

If we consider an M5 cone brane, we might obtain $d=6$ chiral SCFTs dual to $\mathrm{M}$ theory compactified on Einstein 4-manifolds admitting Killing spinors. There are only two cases: $\mathcal{N}=1,2$, although strictly speaking for $\mathcal{N}=1$ we will have to consider orbifolds. The corresponding superconformal algebras [62] have bosonic subalgebra

$$
\mathfrak{s o}_{6,2} \times \mathfrak{s p}_{\mathcal{N}}
$$

with the fermions in the real representation $[(\underline{\mathbf{8}}, \underline{\mathbf{2} \mathcal{N}})]$, where we have introduced the notation $[R]$ to mean the underlying real representation of a complex representation $R$ with a real structure; in other words, $[R] \otimes_{R} \mathbb{C} \cong R$. In this case $(\underline{\boldsymbol{8}}, \underline{\mathbf{2} \mathcal{N}})$ has a real structure by virtue of both $\underline{\mathbf{8}}$ and $\underline{2 \mathcal{N}}$ being quaternionic representations of $\mathfrak{s o}_{6,2}$ and $\mathfrak{s p}_{\mathcal{N}}$, respectively. We recognise the $\mathfrak{s o}_{6,2}$ factor as the isometry algebra of anti-de Sitter space. Now we discuss the other factor.

\subsection{1 $\mathcal{N}=2$}

In this case, the 4-manifold is the round sphere $S^{4}$. The Killing spinors transform as spinors of $\mathfrak{s o}_{5} \cong \mathfrak{s p}_{2}$, in agreement with the structure of the superconformal algebra. 


\subsection{2 $\mathcal{N}=1$}

This case is obtained by considering orbifolds of the previous case. Hence $X_{4} \cong S^{4} / \Gamma$ where $\Gamma$ is an $\mathrm{ADE}$ subgroup of the 'hyperkähler' $\mathrm{Sp}_{1} \subset \mathrm{SO}_{4} \subset$ $\mathrm{SO}_{5}$. Since the centraliser of its Lie algebra $\mathfrak{s p}_{1}$ in $\mathfrak{s o}_{5}$ is precisely the other $\mathfrak{s p}_{1}$ in the decomposition $\mathfrak{s o}_{4} \cong \mathfrak{s p}_{1} \times \mathfrak{s p}_{1}$, the resulting theory has an $\mathfrak{s p}_{1}$ factor in its bosonic subalgebra just as expected. To see how this acts on the spinors, we must decompose the spinor representation $\underline{\mathbf{4}}$ of $\mathfrak{s o}_{5} \cong \mathfrak{s p}_{2}$ in terms of its maximal $\mathfrak{s p}_{1} \times \mathfrak{s p}_{1}$ subalgebra. We find

$$
\underline{\mathbf{4}} \rightarrow(\underline{\mathbf{2}}, \underline{\mathbf{1}}) \oplus(\underline{\mathbf{1}}, \underline{\mathbf{2}}) ;
$$

whence the $\Gamma$-invariant spinors transform according to the $\underline{\mathbf{2}}$ of $\mathfrak{s p}_{1}$, in agreement with the structure of the superconformal algebra. (Notice that the $\underline{\mathbf{2}}$ of $\mathfrak{s p}_{1}$ is indeed quaternionic.)

\section{Conclusions}

We have discussed spaces of the form adS $\mathrm{a}_{p+2} \times X_{d}$ and given a classification of the geometries of $X_{d}$ that admit any given number of Killing spinors. The geometries admitting Killing spinors are, up to quotients: spherical, EinsteinSasaki in dimension $2 k+1,3$-Sasaki in dimension $4 k+3,7$-dimensional manifolds of weak $G_{2}$ holonomy and 6-dimensional nearly Kähler manifolds. When the space $\operatorname{adS}_{p+2} \times X_{d}$ is a solution of a supergravity theory, our analysis gives the amount of supersymmetry the solution preserves. Our approach was based on considering the corresponding brane solution which interpolates between an adS $\mathrm{S}_{p+2} \times X_{d}$ near-horizon geometry and the solution $M_{p+1} \times C(X)$ which is a product of $p+1$-dimensional Minkowski space and the cone $C(X)$ over $X$. The Killing spinors on $X$ are related to parallel spinors on $C(X)$, and the number of these depends only on the holonomy of $C(X)$. In this way, we are able to relate the number of supersymmetries of the two asymptotic regions to each other and to the number of supersymmetries of the brane solution that interpolates between them. We have found the anti-de Sitter supergroup of symmetries that emerges for each supergravity solution.

The brane picture leads to a conjectured duality between the string or $M$ theory on adS $\mathrm{an}_{p+2} \times X_{d}$ and a superconformal field theory with the same supergroup, which we propose arises as the world-volume theory of $p+1$ branes located at the conical singularity of $M_{p+1} \times C(X)$. The geometry then gives rise to some interesting predictions for the properties of the superconformal field theory duals. We finish with some speculations concerning these dual field theories. 
In [56], the IIB theory on adS $S_{5} \times X_{5}$ was considered, where $X_{5}$ is a coset space whose cone $C\left(X_{5}\right)$ is a conically singular Calabi-Yau threefold, and a dual super Yang-Mills theory was proposed, representing D3-branes at the conical singularity. Since this Calabi-Yau space has a description as a Kähler quotient, the quantum field theory was defined as one whose moduli space is this quotient. In other words the Calabi-Yau space is identified as the Higgs branch of the Yang-Mills theory. In particular, since the CalabiYau space is Kähler, the Yang-Mills theory must have four-dimensional $\mathcal{N}=1$ supersymmetry.

Many of the conical geometries we have considered also arise from quotient constructions, and this suggests a similar structure in the field theory duals. For example, for the M2-branes, the cones $C(X)$ are eight-dimensional spaces with $\mathrm{Sp}_{2}, \mathrm{SU}_{4}$ or $\mathrm{Spin}_{7}$ holonomy. Of the examples discussed in section five, all the cones of $\mathrm{Sp}_{2}$ holonomy have a description as hyperkähler quotients [49] and at least some (perhaps all) of those with $\mathrm{SU}_{4}$ holonomy have descriptions as Kähler quotients. Similarly, most of the cones with $\mathrm{SU}_{3}$ holonomy are Kähler quotients.

We propose, in the cases in which $C(X)$ is obtained via a quotient construction in which a space $M$ is 'dividedt' by a group $G$, that the corresponding superconformal field theories have scalars taking values in $M$, and that there is a scalar potential whose space of minima, i.e., the moduli space of the theory, is precisely the cone $C(X)$. It is natural to identify $G$ as the gauge group of the theory. In the (hyper-)Kähler quotient, $M$ is essentially the zero level set of the moment map defined by $G$. This generalises the construction of [56].

For example, consider the 3-dimensional case. Recall that the M2-brane theory arises as an infrared fixed point of the $d=3$ super Yang-Mills theory that is the world-volume theory of the D2-brane. Consider those (nonmaximally supersymmetric) 3-dimensional superconformal field theories that arise as infrared fixed points of $d=3$ super Yang-Mills theories, and which are also the world-volume theories for M2-branes on cones with $\mathrm{Sp}_{2}$ or $\mathrm{SU}_{4}$ holonomy. The quotients are hyperkähler or Kähler, and the $d=3$ Yang-Mills theories will have $\mathcal{N}=4$ or $\mathcal{N}=2$ supersymmetry, respectively. We can choose a potential whose zeroes define a moduli space which is precisely the cone $C(X)$, and a natural proposal is that these Yang-Mills theories flow in the infrared to superconformal field theories which are holographically dual to $M$ theory on $\operatorname{adS}_{4} \times X_{7}$.

The geometries we have found point to a number of situations in which the superconformal field theory dual would be particularly interesting to un- 
derstand. For the M2-branes with $X_{7}$ 3-Sasakian, the dual theory should be some theory with $\mathcal{N}=3$ superconformal symmetry, which would be interesting to construct directly.

We saw in section 5 that any 3-Sasakian 7-manifold may be squashed metrically to give a second Einstein metric with weak $G_{2}$ holonomy. Let $g$ be the 3 -Sasakian metric and $\tilde{g}$ the associated squashed metric on the 7-manifold $X_{7}$. Then $\mathrm{M}$ theory compactified on $\left(X_{7}, g\right)$ has $\mathcal{N}=3$ supersymmetry in four dimensions (or $\mathcal{N}=8$ supersymmetry for the round sphere), while it has $\mathcal{N}=1$ when compactified on $\left(X_{7}, \tilde{g}\right)$. When $g$ is the round metric on the 7 sphere, the phenomenon of squashing was interpreted in the Kaluza-Klein literature [24] as spontaneous (super)symmetry breaking. One can of course give a similar interpretation for any $X_{7}$ which admits a 3-Sasakian metric. From the two $M$ theory compactifications specified by $g$ and $\tilde{g}$ the adS/CFT correspondence gives rise to two corresponding superconformal field theories in three dimensions. It is natural to expect a relation between this pair of SCFTs. The squashing of the 3-Sasakian metric to the weak $G_{2}$ holonomy metric is a continuous deformation, but the resulting one-parameter family of metrics is Einstein only at two points. Of this one-dimensional family of metrics, only two are solutions to $M$ theory. This suggests a corresponding one-parameter family of supersymmetric field theories which is superconformal at only two special values of the parameter, with $\mathcal{N}=3$ (or $\mathcal{N}=8$ ) superconformal symmetry at one point and $\mathcal{N}=1$ superconformal symmetry at the other. Understanding the precise relation between this pair of superconformal field theories should also prove interesting mathematically, since the moduli space of the $\mathcal{N}=1$ theory apparently has $\operatorname{Spin}_{7}$ holonomy.

\section{Acknowledgements}

It is a pleasure to thank Krzysztof Galicki, Alexandros Kehagias, Igor Klebanov and Edward Witten for useful correspondence, and Mohab Abou Zeid for a careful reading of the manuscript. The work of BSA is funded by a PPARC Postdoctoral Fellowship, that of JMF by an EPSRC PDRA, that of CMH by an EPSRC Senior Fellowship and that of BS by an EPSRC Advanced Fellowship. We would like to take this opportunity to thank the respective research councils for their continued support. 


\section{References}

[1] O Aharony, Y Oz, and Z Yin, $M$ theory on $A d S_{p} \times S^{11-p}$ and superconformal field theories, hep-th/9803051.

[2] C Ahn, K Oh, and R Tatar, Orbifolds of $A d S_{7} \times S^{4}$ and six dimensional $(0,1)$ SCFT, hep-th/9804093.

[3] S Aloff and NR Wallach, An infinite family of distinct 7-manifolds admitting positively curved riemannian structures, Bull. Am. Math. Soc. 81 (1975), 93-97.

[4] C Bär, Real Killing spinors and holonomy, Comm. Math. Phys. 154 (1993), 509-521.

[5] E Bergshoeff and JP van der Schaar, On M9-branes, hep-th/9806069.

[6] M Berkooz, A supergravity dual of a $(1,0)$ field theory in six dimensions, hep-th/9802195.

[7] AL Besse, Einstein manifolds, Springer-Verlag, 1987.

[8] R Bielawsi and AS Dancer, The geometry and topology of toric hyperkähler manifolds, MPI and McMaster preprint, 1996.

[9] R Bielawski, Betti numbers of 3-Sasakian quotients of spheres by tori, MPI preprint, 1997.

[10] CP Boyer and K Galicki, 3-Sasakian Manifolds, preprint, 1998.

[11] CP Boyer, K Galicki, and BM Mann, Quaternionic reduction and Einstein manifolds, Comm. Anal. Geom. 1 (1993), 1-51.

[12] _ The geometry and topology of 3-Sasakian manifolds, J. reine angew. Math. 455 (1994), 183-220.

[13] CP Boyer, K Galicki, BM Mann, and E Rees, Compact 3-Sasakian 7manifolds with arbitrary second Betti number, Invent. math. 131 (1998), $321-344$.

[14] L Castellani, A Ceresole, R D'Auria, S Ferrara, P Fré, and M Trigiante, $G / H$ M-branes and adS $_{p+2}$ geometries, hep-th/9803039.

[15] L Castellani, R D'Auria, and P Fré, $\mathrm{SU}(3) \times \mathrm{SU}(2) \times \mathrm{U}(1)$ from $d=11$ supergravity, Nucl. Phys. B239 (1984), 610-652.

[16] L Castellani and LJ Romans, $N=3$ and $N=1$ supersymmetry in a new class of solutions for $d=11$ supergravity, Nucl. Phys. B238 (1984), 683. 
[17] L Castellani, LJ Romans, and NP Warner, A classification of compactifying solutions for $d=11$ supergravity, Nucl. Phys. B241 (1984), 429462.

[18] J Cheeger and D Ebin, Comparison theorems in Riemannian Geometry, North-Holland, Amsterdam, 1975.

[19] E Cremmer, B Julia, and J Scherk, Supergravity in eleven dimensions, Phys. Lett. 76B (1978), 409-412.

[20] R D'Auria, P Fré, and $\mathrm{P}$ van Nieuwenhuizen, $N=2$ matter coupled to supergravity from compactifications on a coset $G / H$ possessing an additional Killing vector, Phys. Lett. 136B (1984), 347-353.

[21] MJ Duff, GW Gibbons, and PK Townsend, Macroscopic superstrings as interpolating solitons, Phys. Lett. 332B (1994), 321-328, hep-th/9405124.

[22] MJ Duff, H Lü, and CN Pope, $\mathrm{AdS}_{5} \times S^{5}$ untwisted, hep-th/9803061.

[23] MJ Duff, H Lü, CN Pope, and E Sezgin, Supermembranes with fewer supersymmetries, Phys. Lett. 371B (1996), 206-214, hep-th/9511162.

[24] MJ Duff, BEW Nilsson, and CN Pope, Kaluza-Klein supergravity, Phys. Rep. 130 (1986), 1-142.

[25] MJ Duff and KS Stelle, Multi-membrane solutions of $D=11$ supergravity, Phys. Lett. 253B (1991), 113-118.

[26] R Entin and J Gomis, Spectrum of chiral operators in strongly coupled gauge theories, hep-th/9804060.

[27] JM Figueroa-O'Farrill, Near-horizon geometries of supersymmetric branes, hep-th/9807149.

[28] T Friedrich, A remark on the first eigenvalue of the Dirac operator on 4-dimensional manifolds, Math. Nach. 102 (1981), 53-56.

[29] T Friedrich and I Kath, Eintein manifolds of dimension five with small eigenvalue of the Dirac operator, J. Diff. Geom. 29 (1989), 263-279.

[30] — 7-dimensional compact riemannian manifolds with Killing spinors, Comm. Math. Phys. 133 (1990), 543-561.

[31] T Friedrich, I Kath, A Moroianu, and U Semmelmann, On nearly parallel $G_{2}$-structures, J. Geom. Phys. 23 (1997), 259-286. 
[32] K Galicki and S Salamon, On Betti numbers of 3-Sasakian manifolds, Geom. Ded. 63 (1996), 45-68.

[33] S Gallot, Equations différentielles caractéristiques de la sphère, Ann. Sci. École Norm. Sup. 12 (1979), 235-267.

[34] JP Gauntlett, GW Gibbons, G Papadopoulos, and PK Townsend, Hyper-Kähler manifolds and multiply intersecting branes, Nucl. Phys. B500 (1997), 133-162, hep-th/9702202.

[35] GW Gibbons and PK Townsend, Vacuum interpolation in supergravity via p-branes, Phys. Rev. Lett. 71 (1993), 3754-3757, hep-th/9302049.

[36] J Gomis, Anti-de Sitter geometry and strongly coupled gauge theories, hep-th/9803119.

[37] A Gray, Kähler submanifolds of homogeneous almost hermitian manifolds, Tôhoku Math. J 21 (1969), 190-194.

[38] —_, Nearly Kähler manifolds, J. Diff. Geom. 4 (1970), 283-309.

[39] _ Weak holonomy groups, Math. Z. 123 (1971), 290-300.

[40] _ Riemannian manifolds with geodesic symmetries of order 3, J. Diff. Geom. 7 (1972), 343-369.

[41] _ The structure of nearly Kähler manifolds, Math. Ann. 223 (1976), 233-248.

[42] A Gray and LM Hervella, The sixteen classes of almost Hermitian manifolds and their linear invariants, Annali di Mat. Pura Appl. (IV) 123 (1980), 35-58.

[43] DJ Gross and MJ Perry, Magnetic monopoles in Kaluza-Klein theories, Nucl. Phys. B226 (1983), 29.

[44] R Grunewald, Six-dimensional manifolds with a real Killing spinor, Ann. Global Anal. Geom. 8 (1990), 43-59.

[45] SS Gubser, Einstein manifolds and conformal field theories, hep-th/9807164.

[46] SS Gubser, IR Klebanov, and AM Polyakov, Gauge theory correlators from noncritical string theory, Phys. Lett. 428B (1998), 105, hep-th/9802109.

[47] R Güven, Black p-brane solutions of $D=11$ supergravity theory, Phys. Lett. 276B (1992), 49-55. 
[48] R Haag, JT Łopuszański, and M Sohnius, All possible generators of supersymmetries of the S-matrix, Nucl. Phys. B88 (1975), 257-274.

[49] NJ Hitchin, A Karlhede, U Lindström, and M Roček, Hyperkähler metrics and supersymmetry, Comm. Math. Phys. 108 (1987), 535-589.

[50] GT Horowitz and A Strominger, Black strings and p-branes, Nucl. Phys. B360 (1991), 197-209.

[51] CM Hull, Exact pp wave solutions of eleven-dimensional supergravity, Phys. Lett. 139B (1984), 39.

[52] — Gravitational duality, branes and charges, Nucl. Phys. B509 (1998), 216-251, hep-th/9705162.

[53] N Itzhaki, JM Maldacena, J Sonnenschein, and S Yankielowicz, Supergravity and the large $N$ limit of theories with sixteen supercharges, hep-th/9802042.

[54] S Kachru and E Silverstein, 4d conformal field theories and strings on orbifolds, hep-th/9802183.

[55] A Kehagias, New type IIB vacua and their F-theory interpretation, hep-th/9805131.

[56] IR Klebanov and E Witten, Superconformal field theory on threebranes at a Calabi-Yau singularity, hep-th/9807080.

[57] M Kreck and S Stolz, A diffeomorphism classification of 7-dimensional homogeneous Einstein manifolds with $\mathrm{SU}(3) \times \mathrm{SU}(2) \times \mathrm{U}(1)$-symmetry, Ann. of Math. 127 (1988), 373-388.

[58] A Lawrence, N Nekrasov, and C Vafa, On conformal field theories in four dimensions, hep-th/9803015.

[59] JM Maldacena, The large $N$ limit of superconformal field theories and supergravity, hep-th/9711200.

[60] A Moroianu, On the infinitesimal isometries of manifolds with Killing spinors, Humboldt Universität preprint.

[61] DR Morrison, Non-spherical horizons, Talk at Strings '98.

[62] W Nahm, Supersymmetries and their representations, Nucl. Phys. B135 (1978), 149-166.

[63] Y Oz and J Terning, Orbifolds of $A d S_{5} \times S^{5}$ and $4 d$ conformal field theories, hep-th/9803167. 
[64] CN Pope and NP Warner, Two new classes of compactifications of $d=11$ supergravity, Class. \& Quantum Grav. 2 (1985), L1-L6.

[65] LJ Romans, New compactifications of chiral $N=2, d=10$ supergravity, Phys. Lett. 153B (1985), 392.

[66] S Salamon, Riemannian geometry and holonomy groups, Pitman Research Notes in Mathematics, no. 201, Longman Scientific \& Technical, 1989.

[67] S Sasaki, On differentiable manifolds with certain structures which are closely related to almost contact structures, Tôhoku Math. J 2 (1960), 459-476.

[68] RD Sorkin, Kaluza-Klein monopole, Phys. Rev. Lett. 51 (1983), 87-90.

[69] S Sulanke, Der erste Eigenwert des Dirac-Operators auf $S^{5} / \Gamma$, Math. Nach. 99 (1980), 259-271.

[70] MY Wang, Parallel spinors and parallel forms, Ann. Global Anal. Geom. 7 (1989), no. 1, 59-68.

[71] E Witten, Anti de sitter space and holography, hep-th/9802150.

[72] _ Baryons and branes in anti-de Sitter space, hep-th/9805112.

[73] (1981), 412-428.

[74] JA Wolf, Spaces of constant curvature, Third ed., Publish or Perish, Boston, 1974. 